\title{
Simulation of pressure drop for combined tapered and non- tapered die for polypropylene using ansys Polyflow
}

\author{
Arman Mohammed Abdalla Ahmed ${ }^{(1)}$, Mohammed Deen Hussein Mohammed \\ (2), Ahmed Ibrahim Ahmed Sidahmed Ahmed ${ }^{(3)}$ \\ ${ }^{(1)(2)(3)}$ Department of Plastic Engineering, School of Engineering \& Technology Industries, College of \\ Engineering, Sudan University of Science and Technology, Khartoum P. O. Box: 72, Sudan.
}

\begin{abstract}
: the pressure drop in combined sections tapered and circular for the flow of polypropylene were studied both analytical and simulation under isothermal and no-slip condition in the die wall. The predicted pressure drop values are compared with three-dimensional (3D) finite element simulation to identify effect of contraction angles, die land and radius on pressure drop. The governing equation of pressure drop was first derived to angle of tapered section, for circular section the pressure drop was studied using different die lands and radii. The three parameters were varied in the ansys Polyflow at specified polymer flow rate and the results are compared with analytical results. For the tapered section the best angle is $\mathbf{4 5}^{-}$analytically and $\mathbf{4 5}^{\circ} \sim \mathbf{5 0} 0^{\circ}$ for the simulation. For circular section of the die the results for die lands variations are almost the same but when varying the varying the radius the results differ at radii less than $2 \mathrm{~cm}$ and approach each other at $2 \mathrm{~cm}$ and above.
\end{abstract}

Index Terms: Tapered die, circular section, pressure drop, Polyflow.

\section{Introduction}

Tapered die is very important in polymer processing, such a profile extrusion, film blowing and tube. The calculation of pressure drop for polymer melt flow through it, is important to the plastic engineering. The theoretical Pressure drop for non -tapered die as function of shear stresses but in tapered the tensile stresses will be set up in the fluid and their effects superimposed on the effects due to shear stresses this problem was analyzed for the flow fluid along coni-cylindrical. The flow is influenced by three factors Shear, Extensional and Entrance effects.

The derivation of equations of tapered and non-tapered dies assumed the polymer melt, is a Newtonian, isothermal, uncompressible and no slip on the die wall. [1]

The pressure drop for Non-tapered circular die

$$
\Delta p_{\text {circular }}=\frac{2 L_{1} \tau_{1}}{R_{1}}
$$

Where:

$R_{1}$ : Radius of the die channel, $\mathrm{L}_{1}$ : length of the die land, $\Delta p_{\text {circular }}$

: Pressure drop over the circular channel

The pressure drop for Tapered circular die

i.

Pressure Drop Due to Shear, $\Delta p_{s}$

$$
\Delta p_{s}=\frac{2 \tau_{1}}{3 \tan \theta}\left(1-\left(\frac{R_{1}}{R_{2}}\right)^{\mathrm{a}}\right)
$$

$\tau_{1}=\mu_{1} \cdot \gamma_{1}$

Where:

$\tau_{1} \quad$ Shear stress, $\tilde{\gamma}_{1} \quad$ Shear rate, $\mu_{1}$ Shear viscosity

ii. Pressure Drop Due to Extensional Flow $\Delta p_{E}$

$$
\begin{aligned}
& \Delta p_{E}=\frac{2 \sigma_{1}}{3}\left(1-\left(\frac{R_{1}}{R_{2}}\right)^{\mathrm{a}}\right) \\
& \sigma_{1}=\lambda \frac{\tan (\theta)}{3}\left(\frac{4 Q}{\pi R_{1}{ }^{\mathrm{a}}}\right)=\lambda \frac{\tan (\theta)}{3} \tilde{\gamma}_{1}=\lambda \varepsilon_{1}^{\mathrm{z}}
\end{aligned}
$$

Where :

$\lambda$ tensile viscosity about three time of shear viscosity at low shear rate, $\frac{\varepsilon}{\varepsilon}$ tensile strain. 


\section{iii. Pressure Drop at Die Entrance, $\Delta \mathbf{P}_{2}$}

$$
\Delta P_{2}=\frac{2 \sqrt{2}}{3}\left(\frac{4 Q}{\pi R_{2}{ }^{a}}\right)\left(\mu_{2} \lambda\right)^{1 / 2}
$$

When the fluid enters the die from a reservoir it will conform to a streamline shape such that the pressure drop is a minimum. This will tend to be of a coni-cylindrical geometry and the pressure drop, $\Delta P_{2}$, may be estimated by considering an infinite number of very short frustums of a cone. [1]

The above mentioned study use the assumption of no-slip at the solid boundary, However, polymer melts can slip at solid interface when the wall shear stress exceeds a critical value so that there approximate analytical equations that are derived for the calculation of pressure drop of power-law fluids for viscous flow through tapered dies for a wide range of wall-slip conditions and the predicted pressure drop values are compared with two-dimensional (2D) finite element calculations to identify contraction angles for which the analytical equations can be used.[2]

Entrance pressure drop is a large one when a molten polymer flows through a Tapered die of a given angle[3] .This pressure is required in order to calculate the true shear stress in capillary flow and also frequently the apparent extensional rheology of molten polymers, a method well practiced in industry. Therefore, it is important to understand the origin of this excess pressure and consequently to be able to predict it. [4]

The Entrance pressure drop as a function of contraction angle at a given apparent shear rate under slip or no-slip boundary conditions. This was studied for a branched polypropylene (PP) melt both experimentally and theoretically. The entrance pressure was first determined experimentally as a function of the contraction angle ranging from $10^{\circ}$ to $150^{\circ}$. It was found that at a given apparent shear rate, the pressure loss decreases with increasing contraction angle from $10^{\circ}$ to about $45^{\circ}$, and consequently slightly increases from $45^{\circ}$ up to contraction angles of $150^{\circ}$.[5]

The Entrance pressure drop in the capillary flow of several types of polyethylene were studied both experimentally and numerically under slip and no-slip conditions. These losses were first measured as a function of the contraction angle ranging from $15^{\circ}$ to $90^{\circ}$. It was found that the excess pressure loss attains a local minimum at a contraction angle of about $30^{\circ}$ for all types of polyethylene examined.[4]

It is the main objective of this work to study the pressure drop in polymer extrusion die combined of two sections tapered and circular (see Fig.1) analytical and three-dimensional Numerical simulation with ansys Polyflow software as a functions of entrance angle, die land and radius for polypropylene material .

Numerical simulation has the potential to uncover important interior details of the extrusion process such as velocity, shear stress, pressure, and temperature fields in the region of interest, which is not possible to do experimentally.[6]

The carried research and obtained data will be the basis of modeling of extrusion process using Ansys Polyflow program. It enables simulation flow viscous and viscoelastic behavior. This program use equations of conservation of mass, momentum and energy, and also of various rheological models describing material properties and behavior during processing. [7]

\section{Materials And Methods}

Materials: In previews work the best viscosity model (Carreau-Yasuda law) was estimated (viscosity versus shear rate) at isothermal condition for experimental data obtained from melt flow index tester for Polypropylene after fitted data in ansys Polyflow (polymat) and applied statistical analysis method of Percentage Root Mean Square Error (\%PRMSE).[8]

Where

$$
\mu=\mu_{\mathrm{mos}}+\left(\mu_{0}-\mu_{\mathrm{max}}\right)\left(1+\left(\lambda_{\tilde{y}}\right)^{\alpha}\right)^{\frac{n-1}{\alpha}}
$$

$\mu_{\mathrm{sec}}=$ infinite-shear-rate viscosity $=0.01445808$ poise

$\mu_{0}=$ zero-shear-rate viscosity $=67380.02$ poise

$\lambda=$ natural time (i.e., inverse of the shear rate at which the fluid changes from Newtonian to power-law behavior) $=0.03531332 \mathrm{sec}$

$a=$ index that controls the transition from the Newtonian plateau to the power-law region $=0.4534786$

$\mathrm{n}=$ power-law index $=0.5375814 \mathrm{E}-05$

Analytically: The Die combined with non- Tapered circular section and Tapered circular section shown in Fig (1).The total pressure drop in die combination of two section Eq (1 to 6) at the steady -state the Flow rate constant the total pressure drop is:

$$
\begin{gathered}
\Delta p_{\text {Total }}=\Delta p_{\text {circular }}+\Delta p_{s}+\Delta p_{E}+\Delta p_{2} \\
\Delta p_{\text {Total }}=\frac{2 L_{1} \tau_{1}}{R_{1}}+\frac{2 \tau_{1}}{3 \tan \theta}\left(1-\left(\frac{R_{1}}{R_{2}}\right)^{\mathrm{a}}\right)+\frac{2 \sigma_{1}}{3}\left(1-\left(\frac{R_{1}}{R_{2}}\right)^{\mathrm{a}}\right)+\frac{2 \sqrt{2}}{3}\left(\frac{4 Q}{\pi R_{2}^{\mathrm{a}}}\right)\left(\mu_{2} \lambda\right)^{1 / 2}
\end{gathered}
$$


Where:

$R_{1}=0.5 \mathrm{~cm}, L_{1}=1 \mathrm{~cm}, \mu_{1}=$ from $\mathrm{Eq}(7), \tau_{1}=\mu_{1} \times \gamma_{1}^{*}=\mu_{1} \times \frac{4 Q}{\pi R_{1}{ }^{3}}$

$\mathrm{R}_{2}=3.1 \mathrm{~cm}_{s} \lambda=3 \times \mu_{1}, \varepsilon_{1}^{*}=\frac{\dot{\gamma}_{1}}{3} \tan (\theta), \sigma_{1}=\lambda \varepsilon_{1}^{*}, \mathrm{Q}=5 \frac{\mathrm{cm}^{d}}{\mathrm{~s}}$

Analytical Effect of angle on Pressure Drop: The optimum angle for design when pressure drop is minimum for derivative $\mathrm{Eq}(8)$ :

$$
\begin{aligned}
& \frac{d \Delta p_{\text {Total }}}{d \theta}=-\frac{2 \tau_{1}}{a}\left(\frac{1+\tan ^{2} \theta}{\tan ^{2} \theta}\right)\left(1-\left(\frac{R_{1}}{R_{2}}\right)^{a}\right)+\frac{2 \lambda\left(\frac{\tilde{z}_{1}}{3}\left(1+\tan ^{2} \theta\right)\right.}{a}\left(1-\left(\frac{R_{1}}{R_{2}}\right)^{a}\right)=0 \\
= & -\frac{2 \tau_{1}}{3}\left(\frac{1+\tan ^{2} \theta}{\tan ^{2} \theta}\right)\left(1-\left(\frac{R_{1}}{R_{2}}\right)^{a}\right)+\frac{2 \frac{\lambda}{3}\left(\dot{\gamma}_{1}\left(1+\tan ^{2} \theta\right)\right)}{3}\left(1-\left(\frac{R_{1}}{R_{2}}\right)^{a}\right)=0 \\
= & -\frac{2 \mu_{1} \dot{\gamma}_{1}}{3}\left(\frac{1+\tan ^{2} \theta}{\tan ^{2} \theta}\right)\left(1-\left(\frac{R_{1}}{R_{2}}\right)^{\mathrm{a}}\right)+\frac{2 \mu_{1} \hat{\gamma}_{1}\left(\left(1+\tan ^{2} \theta\right)\right)}{3}\left(1-\left(\frac{R_{1}}{R_{2}}\right)^{\mathrm{a}}\right)=0 \\
\left(\frac{1+\tan ^{2} \theta}{\tan ^{2} \theta}\right)=\left(1+\tan ^{2} \theta\right) & \tan ^{2} \theta=1 \therefore \theta=45
\end{aligned}
$$

Calculate the values of pressure drop by applying Eq (8) at different angles $(10,20,30,40,45,50,60,70$ and $80^{\circ}$ ) when die radius $0.5 \mathrm{~cm}$ and land $1 \mathrm{~cm}$ shown in the Table (1) and Fig (3).

Analytical Effect of Die land on Pressure Drop: The relationship between pressure drop and die land can be derived from $\mathrm{Eq}(8)$ :

$$
\frac{d \Delta p_{\text {Total }}}{d L_{1}}=\frac{2 \tau_{1}}{R_{1}}
$$

Calculate the values of pressure drop by applying Eq (8) at different die land $(0.5,1,2$, and $5 \mathrm{~cm})$ shown in the table (3) and Fig (5).

Analytical Effect of Die radius on Pressure Drop: The relationship between pressure drop and die radius can be derived from $\mathrm{Eq}(8)$ :

$$
\begin{aligned}
& \Delta \mathrm{p}_{\text {Total }}=\frac{2 \mathrm{~L}_{1} \tau_{1}}{\mathrm{R}_{1}}+\frac{2 \tau_{1}}{3 \tan \theta}\left(1-\left(\frac{\mathrm{R}_{1}}{\mathrm{R}_{2}}\right)^{\mathrm{a}}\right)+\frac{2 \sigma_{1}}{3}\left(1-\left(\frac{\mathrm{R}_{1}}{\mathrm{R}_{2}}\right)^{\mathrm{a}}\right)+\frac{2 \sqrt{2}}{3}\left(\frac{4 \mathrm{Q}}{\pi \mathrm{R}_{2}{ }^{a}}\right)\left(\mu_{2} \lambda\right)^{1 / 2}---(8) \\
& \Delta \mathrm{p}_{\text {Total }}=\frac{2 \mathrm{~L}_{1} \mu_{1}}{\mathrm{R}_{1}}\left(\frac{4 \mathrm{Q}}{\pi \mathrm{R}_{1}^{a}}\right)+\frac{2 \mu_{1}}{3 \tan \theta}\left(\frac{4 \mathrm{Q}}{\pi \mathrm{R}_{1}^{\mathrm{a}}}\right)\left(1-\left(\frac{\mathrm{R}_{1}}{\mathrm{R}_{2}}\right)^{\mathrm{a}}\right)+\frac{2 \lambda}{9}\left(\frac{4 \mathrm{Q}}{\pi \mathrm{R}_{1}^{a}}\right)\left(1-\left(\frac{\mathrm{R}_{1}}{\mathrm{R}_{2}}\right)^{\mathrm{a}}\right) \\
& +\frac{2 \sqrt{2}}{3}\left(\frac{4 Q}{\pi R_{2}}\right)\left(\mu_{2} \lambda\right)^{1 / 2} \\
& \Delta \mathrm{p}_{\text {Total }}=\frac{8 \mathrm{QL} \mathrm{L}_{1} \mu_{1}}{\pi}\left(\frac{1}{\mathrm{R}_{1}^{4}}\right)+\frac{8 \mathrm{Q} \mu_{1}}{3 \pi \tan \theta}\left(\frac{1}{\mathrm{R}_{1}^{2}}\right)\left(1-\left(\frac{\mathrm{R}_{1}}{\mathrm{R}_{2}}\right)^{\mathrm{a}}\right)+\frac{8 \lambda \mathrm{Q}}{9 \pi}\left(\frac{1}{\mathrm{R}_{1}^{2}}\right)\left(1-\left(\frac{\mathrm{R}_{1}}{\mathrm{R}_{2}}\right)^{\mathrm{a}}\right) \\
& +\frac{2 \sqrt{2}}{3}\left(\frac{4 Q}{\pi R_{2}^{a}}\right)\left(\mu_{2} \lambda\right)^{1 / 2} \\
& \Delta \mathrm{p}_{\text {Total }}=\frac{8 \mathrm{QL} \mu_{1} \mu_{1}}{\pi}\left(\frac{1}{\mathrm{R}_{1}^{4}}\right)+\frac{8 \mathrm{Q} \mu_{1}}{3 \pi \tan \theta}\left(\frac{1}{\mathrm{R}_{1}^{2}}\right)-\frac{8 \mathrm{Q} \mu_{1}}{3 \pi \tan \theta}\left(\frac{1}{\mathrm{R}_{2}^{2}}\right)+\frac{8 \lambda \mathrm{Q}}{9 \pi}\left(\frac{1}{\mathrm{R}_{1}^{2}}\right)-\frac{8 \lambda \mathrm{Q}}{9 \pi}\left(\frac{1}{\mathrm{R}_{2}^{2}}\right) \\
& +\frac{2 \sqrt{2}}{3}\left(\frac{4 Q}{\pi R_{2}}\right)\left(\mu_{2} \lambda\right)^{1 / 2} \\
& \Delta \mathrm{p}_{\text {Total }}=\frac{8 Q L_{1} \mu_{1}}{\pi}\left(\frac{1}{R_{1}^{4}}\right)+\frac{8 Q \mu_{1}}{3 \pi \tan \theta}\left(\frac{1}{R_{1}^{2}}\right)+\frac{8 \lambda Q}{9 \pi}\left(\frac{1}{R_{1}^{2}}\right)-\frac{8 Q \mu_{1}}{3 \pi \tan \theta}\left(\frac{1}{R_{2}^{2}}\right)-\frac{8 \lambda Q}{9 \pi}\left(\frac{1}{R_{2}^{2}}\right) \\
& +\frac{2 \sqrt{2}}{3}\left(\frac{4 Q}{\pi R_{2}{ }^{a}}\right)\left(\mu_{2} \lambda\right)^{1 / 2} \\
& \Delta \mathrm{p}_{\text {Total }}=C_{1}\left(\frac{1}{\mathrm{R}_{1}^{4}}\right)+C_{2}\left(\frac{1}{\mathrm{R}_{1}^{a}}\right)+C_{a}\left(\frac{1}{\mathrm{R}_{1}^{a}}\right)+C_{4}
\end{aligned}
$$

where 


$$
\begin{aligned}
C_{1}=\frac{8 \mathrm{QL} \mathrm{L}_{1} \mu_{1}}{\pi}, C_{2} & =\frac{8 \mathrm{Q} \mu_{1}}{3 \pi \tan \theta}, C_{\mathrm{a}}=\frac{8 \lambda \mathrm{Q}}{9 \pi}, C_{4} \\
& =-\frac{8 \mathrm{Q} \mu_{1}}{3 \pi \tan \theta}\left(\frac{1}{\mathrm{R}_{2}{ }^{\mathrm{a}}}\right)-\frac{8 \lambda \mathrm{Q}}{9 \pi}\left(\frac{1}{\mathrm{R}_{2}{ }^{\mathrm{a}}}\right)+\frac{2 \sqrt{2}}{3}\left(\frac{4 \mathrm{Q}}{\pi \mathrm{R}_{2}{ }^{\mathrm{a}}}\right)\left(\mu_{2} \lambda\right)^{1 / 2}
\end{aligned}
$$

Calculate the values of pressure drop by applying Eq (8) at different die radii $(0.5,0.6,0.7,0.8,1,1.5 \mathrm{and} 2 \mathrm{~cm})$ shown in the table (5) and Fig (7).

\section{Simulation model:}

Model: creating a sketch on the XY Plane to half of theoretical model was shown in Fig (2) and set dimensions then revolve the sketch to complete 3D model (see Fig 3).

Meshing: automatically generate medium meshing and assign to three face as boundary input, output and wall.

Setup (Polydata): the task is FEM, steady- state, isothermal, enter materials data (type of viscosity model Eq 7) the boundary set as:

Boundary 1: Input =inflow $\left(5 \mathrm{~cm}^{3} / \mathrm{s}\right)$

Boundary 2: Output=outflow,

Boundary 3: Wall = zero normal velocity and zero surface velocity condition $V_{n}=0 \mathrm{Vs}=0$

Solution and Result: Contours of output parameters (shear rate, pressure drop) are graphically represented at every mesh of the structural geometry.

Generate results for multiple design points for study effect of die dimensions (angle, die land L1 and radius R1) at the values of analytical considered to the parameters (pressure drop was taken between average pressure at Die inlet and average pressure at Die outlet, average velocity, maximum and minimum shear rate) using the parameter and Design Points view. The results shown in Tables (2, 4 and 6) and Figs (3, 5 and 7).

\section{Results And Discussion}

The analytical derivation for the taper angle to obtain minimum pressure drop is $45^{\circ}$ the same result was obtained when calculating the different types of pressure drops for range of taper angle $10^{\circ}$ to $80^{\circ}$. The taper angle don't affect the value of strain shear rate, on the other hand when using poly flow software the taper angle that gives minimum pressure drop is between $45^{\circ} \sim 50^{\circ}$ (see Figs 3). The same result was obtain experimentally by E. Mitsoulis etal[5] .This study shows that the shear strain rate for minimum pressure drop is not affected by taper angle while the software, the shear strain rate varies as the taper angle is increased (see Table (2) and Fig. 4).

The pressure gradient across die land Eq (9) is equal to $2.1769 \times 10^{6}$ dyne. $\mathrm{cm}^{-1}$. The software gives a value of $2.4529 \times 10^{6}$ dyne. $\mathrm{cm}^{-1}$. Both results were obtained when varying die land $0.5,1$, and $5 \mathrm{~cm}$. (see Tables(3),(4) and Fig.5)

When studying the relation between the die radius and pressure drop using both calculation Eq (10) and software the pressure drop is inversely related to die radius.At a radius of $2 \mathrm{~cm}$ and above the results tend to be identical. (see Tables (5),(6) and Fig.6)

\section{Conclusions}

The best taper angle for PP die that consist of circular section and tapered section is $45^{\circ}$.the highest shear rate was at the interface between the circular section and tapered section which gives the highest pressure drop .To prevent this the discontinuity between the two sections should be disappear .

\begin{tabular}{|c|c|c|c|c|c|c|c|c|c|c|c|c|c|c|c|c|}
\hline $\begin{array}{c}R_{1} \\
(\mathrm{~cm})\end{array}$ & $\begin{array}{c}L_{1} \\
(\mathrm{~cm})\end{array}$ & $\begin{array}{c}\text { Angle } \\
(\theta) \\
\text { degree }\end{array}$ & $\begin{array}{c}Q \\
\left(\frac{\mathrm{cm}^{3}}{\mathrm{~s}}\right)\end{array}$ & $\begin{array}{c}\dot{\gamma}_{1} \\
\left(s^{-1}\right)\end{array}$ & $\begin{array}{c}\dot{\gamma}_{2} \\
\left(s^{-1}\right)\end{array}$ & $\left(\begin{array}{c}\mu_{1} \\
\frac{\text { dyne }}{c m^{2}} \cdot s\end{array}\right)$ & $\left(\frac{\begin{array}{c}\mu_{2} \\
\text { dyne }\end{array}}{c m^{2}} \cdot s\right)$ & $\left(\begin{array}{c}\tau_{1} \\
\frac{\text { dyne }}{c m^{2}}\end{array}\right)$ & $\left(\begin{array}{c}\lambda \\
\text { dyne } \\
c m^{2}\end{array} \cdot s\right)$ & $\mid \begin{array}{c}\dot{\varepsilon}_{1} \\
\left(s^{-1}\right)\end{array}$ & $\left(\begin{array}{c}\sigma_{1} \\
\text { dyne } \\
\text { cm }^{2}\end{array}\right)$ & $\left|\begin{array}{c}\Delta p_{\text {circular }} \\
\left(\frac{\text { dyne }}{\text { cm }^{2}}\right)\end{array}\right|$ & $\begin{array}{c}\Delta p_{s} \\
\text { dyne } \\
\left(\frac{c^{2}}{c m^{2}}\right)\end{array}$ & $\begin{array}{c}\Delta p_{E} \\
\left(\frac{d y n e}{c m^{2}}\right)\end{array}$ & $\begin{array}{c}\Delta p_{2} \\
\left(\frac{d y n e}{c m^{2}}\right)\end{array}$ & $\begin{array}{l}\Delta p_{\text {Total }} \\
\text { dyne } \\
\left(\frac{\mathrm{cm}^{2}}{\mathrm{~cm}^{2}}\right)\end{array}$ \\
\hline 0.5 & 1.0 & 10.0 & 5.0 & 50.9 & 0.2 & 10685.7 & 53630.6 & 544216.2 & 32057.0 & 3.0 & 95960.0 & 2176865.02 & 2048969.0 & 63704.9 & 8353.8 & 4297892.7 \\
\hline 0.5 & 1.0 & .0 & 5.0 & 50.9 & 0.2 & 10685.7 & 53630.6 & 544216.2 & 32057.0 & 6.2 & 198078.5 & 2176865.0 & 992632.0 & 131498.3 & 8353.8 & 3309349.1 \\
\hline 0.5 & & & 5.0 & 50.9 & 0.2 & 10685.7 & 5363 & 544216.2 & 32057.0 & 9.8 & & & & 208590.0 & 8353.8 & 78.9 \\
\hline 0.5 & & & 5.0 & 50.9 & 0.2 & & & 544216.2 & & 14.2 & & & & & 8353.8 & \\
\hline 0.5 & & & 5.0 & & 0 & & & & & 17.0 & & & & & 53.8 & \\
\hline 0.5 & & & 5.0 & 50.9 & 0.2 & & 5363 & 544216.2 & 32057.0 & 20.2 & 6485 & 2176 & 57.1 & 430566.9 & 8353.8 & \\
\hline 0.5 & 1 & & 5.0 & 50.9 & 0.2 & 10685.7 & 53630.6 & 544216.2 & 32057.0 & 29.4 & 942610.2 & 2176 & 990.0 & 625770.1 & 8353.8 & 78.9 \\
\hline 0.5 & 1.0 & 0.0 & 5.0 & 50.9 & 0.2 & 10685.7 & 53630.6 & 544216.2 & 32057.0 & 46.6 & 1495221.8 & 21768 & 131498.3 & 992632.0 & 8353.8 & 3309349.1 \\
\hline 0.5 & 1.0 & 80.0 & 5.0 & 50.9 & 0.2 & 10685.7 & 53630.6 & 544216.2 & 32057.0 & 96.3 & 3086403.7 & 2176865.0 & 63704.9 & 2048969.0 & 8353.8 & 4297892.7 \\
\hline
\end{tabular}

Table 1: Calculation pressure drop at different angle 
Table 2: Die design points at different die angle

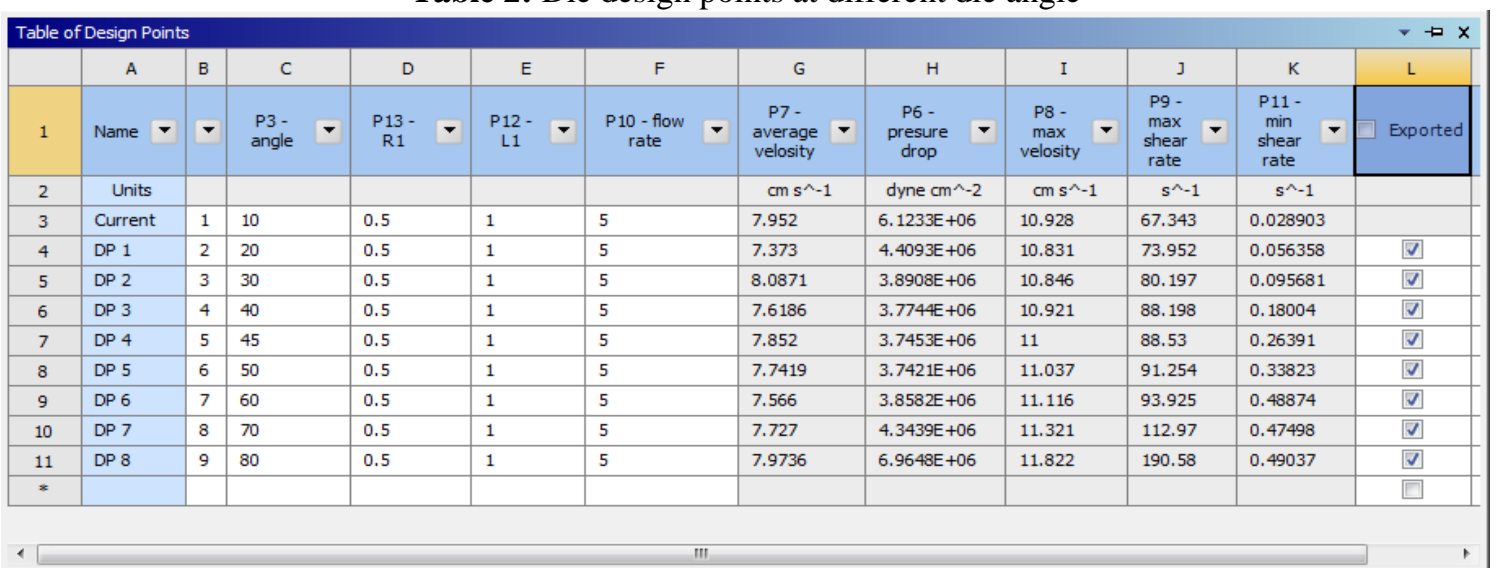

Table 3: Calculation pressure drop at different Die land

\begin{tabular}{|c|c|c|c|c|c|c|c|c|c|c|c|c|c|c|c|c|}
\hline $\begin{array}{c}R_{1} \\
(\mathrm{~cm})\end{array}$ & $\begin{array}{c}L_{1} \\
(\mathrm{~cm})\end{array}$ & $\begin{array}{c}\text { Angle } \\
(\theta) \\
\text { degree }\end{array}$ & $\begin{array}{c}Q \\
\left(\frac{\mathrm{cm}^{3}}{\mathrm{~s}}\right)\end{array}$ & $\begin{array}{c}\dot{\gamma}_{1} \\
\left(s^{-1}\right)\end{array}$ & $\begin{array}{c}\dot{\gamma}_{2} \\
\left(s^{-1}\right)\end{array}$ & $\left(\begin{array}{c}\mu_{1} \\
\left(\frac{\text { dyne }}{c m^{2}} \cdot s\right.\end{array}\right)$ & $\left(\begin{array}{c}\mu_{2} \\
\left(\frac{\text { dyne }}{c m^{2}} \cdot s\right.\end{array}\right)$ & $\left(\begin{array}{c}\tau_{1} \\
\frac{\text { dyne }}{c m^{2}}\end{array}\right)$ & $\left(\begin{array}{c}\lambda \\
\left(\frac{\text { dyne }}{c m^{2}} \cdot s\right.\end{array}\right)$ & $\begin{array}{c}\dot{\varepsilon}_{1} \\
\left(s^{-1}\right)\end{array}$ & $\left(\frac{\left.\begin{array}{c}\sigma_{1} \\
\text { dyne }\end{array}\right)}{c m^{2}}\right)$ & $\left|\begin{array}{c}\Delta p_{\text {circular }} \\
\text { dyne } \\
\left(\frac{\mathrm{cm}^{2}}{2}\right)\end{array}\right|$ & $\begin{array}{c}\Delta p_{s} \\
\text { dyne } \\
\left(\frac{c^{2}}{c m^{2}}\right)\end{array}$ & $\begin{array}{c}\left.\begin{array}{c}\Delta p_{E} \\
d y n e\end{array}\right) \\
\left(\frac{c m^{2}}{c}\right)\end{array}$ & $\begin{array}{c}\Delta p_{2} \\
\left(\frac{d y n e}{c m^{2}}\right)\end{array}$ & $\begin{array}{l}\Delta p_{\text {Total }} \\
\text { dyne } \\
\left(\frac{\text { cm }^{2}}{2}\right)\end{array}$ \\
\hline 0.5 & 0.5 & 45 & 5 & 50.93 & 0.214 & 10685.661 & 53630.599 & $5.44 \mathrm{E}+05$ & 32056.98272 & 16.977 & $5.44 \mathrm{E}+05$ & $1.09 \mathrm{E}+06$ & $3.61 \mathrm{E}+05$ & $3.61 \mathrm{E}+05$ & $8.35 \mathrm{E}+03$ & $1.819 \mathrm{E}+06$ \\
\hline 0.5 & 1 & 45 & 5 & 50.93 & 0.214 & 10685.661 & 53630.599 & $5.44 \mathrm{E}+05$ & 32056.98272 & 16.977 & $5.44 \mathrm{E}+05$ & $2.18 \mathrm{E}+06$ & $3.61 \mathrm{E}+05$ & $3.61 \mathrm{E}+05$ & $8.35 \mathrm{E}+03$ & $2.908 \mathrm{E}+06$ \\
\hline 0.5 & 2 & 45 & 5 & 50.93 & 0.214 & 10685.661 & 53630.599 & $5.44 \mathrm{E}+05$ & 32056.98272 & 16.977 & $5.44 \mathrm{E}+05$ & $4.35 \mathrm{E}+06$ & $3.61 \mathrm{E}+0 \mathrm{~S}$ & $3.61 \mathrm{E}+05$ & $8.35 \mathrm{E}+0$ & $5.085 \mathrm{E}+06$ \\
\hline 0.5 & 5 & 45 & 5 & 50.93 & 0.214 & 10685.661 & 53630.599 & $5.44 \mathrm{E}+05$ & 32056.98272 & 16.977 & $5.44 \mathrm{E}+05$ & $1.09 \mathrm{E}+07$ & $3.61 \mathrm{E}+0$ & $3.61 \mathrm{E}+05$ & $8.35 \mathrm{E}+03$ & $1.162 \mathrm{E}+07$ \\
\hline
\end{tabular}

Table 4: Die design points at different die land

\begin{tabular}{|c|c|c|c|c|c|c|c|c|c|c|c|c|c|c|c|}
\hline \multicolumn{16}{|c|}{ Table of Design Points } \\
\hline & A & B & c & & D & & E & $\mathrm{F}$ & & G & $\mathrm{H}$ & I & J & $\mathrm{K}$ & L \\
\hline 1 & Name & 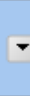 & $\begin{array}{l}\text { P12- } \\
\text { angle }\end{array}$ & $\checkmark$ & $\begin{array}{l}\mathrm{P} 14- \\
\text { L1 }\end{array}$ & $\checkmark$ & $\underset{\mathrm{R} 13-}{\mathrm{P} 13-}$ & $\begin{array}{l}\text { P10 - flow } \\
\text { rate }\end{array}$ & $\checkmark$ & $\begin{array}{l}\text { P7 - } \\
\text { average - } \\
\text { velosity }\end{array}$ & $\begin{array}{c}\text { P6 - } \\
\text { presure } \\
\text { drop }\end{array}$ & $\begin{array}{c}\text { P8 - } \\
\text { max } \\
\text { velosity }\end{array}$ & $\begin{array}{l}\mathrm{P9}- \\
\text { max } \\
\text { shear } \\
\text { rate }\end{array}$ & $\begin{array}{l}\text { P11 - } \\
\text { min } \\
\text { shear } \\
\text { rate }\end{array}$ & $\square$ Exported \\
\hline 2 & Units & & & & & & & & & $\mathrm{cm} \mathrm{s} \mathrm{s}^{\wedge}-1$ & dyne $\mathrm{cm}^{\wedge}-2$ & $\mathrm{~cm} \mathrm{~s}^{\wedge}-1$ & $s^{\wedge}-1$ & $s^{\wedge}-1$ & \\
\hline 3 & Current & 1 & 45 & & 0.5 & & 0.5 & 5 & & 7.8359 & $2.4768 E+06$ & 11.035 & 93.123 & 0.22448 & \\
\hline 4 & DP 1 & 2 & 45 & & 1 & & 0.5 & 5 & & 7.7541 & $3.7479 E+06$ & 11 & 90.442 & 0.24497 & $\nabla$ \\
\hline 5 & DP 2 & 3 & 45 & & 2 & & 0.5 & 5 & & 7.6669 & $6.1754 E+06$ & 11.015 & 91.402 & 0.21087 & $\nabla$ \\
\hline 6 & DP 3 & 4 & 45 & & 5 & & 0.5 & 5 & & 7.5222 & $1.3533 E+07$ & 11.106 & 81.716 & 0.26696 & $\nabla$ \\
\hline$*$ & & & & & & & & & & & & & & & 回 \\
\hline
\end{tabular}

Table 5: Calculation pressure drop at different Die radius

\begin{tabular}{|c|c|c|c|c|c|c|c|c|c|c|c|c|c|c|c|c|}
\hline $\begin{array}{c}R_{1} \\
(\mathrm{~cm})\end{array}$ & $\begin{array}{c}L_{1} \\
(\mathrm{~cm})\end{array}$ & $\begin{array}{c}\text { Angle } \\
(\theta) \\
\text { degree }\end{array}$ & $\begin{array}{c}Q \\
\left(\frac{\mathrm{cm}^{3}}{\mathrm{~s}}\right)\end{array}$ & $\begin{array}{c}\gamma_{1} \\
\left(s^{-1}\right)\end{array}$ & $\begin{array}{c}\dot{\gamma_{2}} \\
\left(s^{-1}\right)\end{array}$ & $\left(\frac{\begin{array}{c}\mu_{1} \\
\text { dyne }\end{array}}{c m^{2}} \cdot s\right.$ & $\begin{array}{c}\mu_{2} \\
\text { dyne } \\
\text { cm }\end{array}$ & $\left(\begin{array}{c}\tau_{1} \\
\frac{\text { dyne }}{c m^{2}}\end{array}\right)$ & $\left(\begin{array}{c}\lambda \\
\left(\frac{\text { dyne }}{c m^{2}} \cdot s\right)\end{array}\right)$ & $\left(\begin{array}{c}\dot{\varepsilon}_{1} \\
\left(s^{-1}\right)\end{array}\right.$ & $\left(\begin{array}{c}\sigma_{1} \\
\text { dyne } \\
\left.\frac{c m^{2}}{c m^{2}}\right)\end{array}\right.$ & $\left|\begin{array}{c}\Delta p_{\text {circular }} \\
\left(\frac{\text { dyne }}{\text { cm }^{2}}\right)\end{array}\right|$ & $\begin{array}{c}\Delta p_{s} \\
\text { dyne } \\
\left(\frac{c^{2}}{c^{2}}\right)\end{array}$ & $\begin{array}{c}\Delta p_{E} \\
\text { dyne } \\
\left(\frac{c^{2} m^{2}}{c^{2}}\right)\end{array}$ & $\begin{array}{c}\Delta p_{2} \\
\text { dyne } \\
\left(\frac{c^{2} m^{2}}{c^{2}}\right)\end{array}$ & $\begin{array}{l}\Delta p_{\text {Total }} \\
\text { dyne } \\
\left(\frac{c^{2}}{c m^{2}}\right)\end{array}$ \\
\hline 0.5 & 1.0 & 45.0 & 5.0 & 50.9 & 0.2 & 10685.7 & 53630.6 & 544216.2 & 32057.0 & 17.0 & 544216.2 & 2176865.0 & 361288.5 & 361288.5 & 8353.8 & 2907795.8 \\
\hline 0.6 & 1.0 & 45.0 & 5.0 & 29.5 & 0.2 & 14321.5 & 53630.6 & 422100.3 & 42964.6 & 9.8 & 422100.3 & 1407000.8 & 279359.9 & 279359.9 & 9671.2 & 1975391.8 \\
\hline 0.7 & 1.0 & 5.0 & 5.0 & 18.6 & 0.2 & 17867.4 & 53630.6 & 331624.9 & 53602.2 & 6.2 & 331624.9 & 947499.7 & 218537.8 & 218537.8 & 10802.3 & 1395377.6 \\
\hline 0.8 & 1.0 & 4 & 5.0 & 12.4 & 0.2 & 21225.3 & 53630.6 & 263915.4 & 63676.0 & 4.1 & 2639 & 659788.6 & 172919.8 & 172919. & 11773.7 & 1017401.8 \\
\hline 1.0 & 1.0 & 45.0 & 5.0 & 6.4 & 0.2 & 27225.7 & 53630.6 & 173324.0 & 81677.0 & 2.1 & 173324.0 & 346648.0 & 111670.7 & 111670.7 & 13334.4 & 583323.7 \\
\hline 1.5 & 1.0 & 45.0 & 5.0 & 1.9 & 0.2 & 38249.0 & 53630.6 & 72148.4 & 114747.1 & 0.6 & 72148.4 & 96197.9 & 42649.8 & 42649.8 & 15805.1 & 197302.6 \\
\hline 2.0 & 1.0 & 45.0 & 5.0 & 0.8 & 0.2 & 45244.9 & 53630.6 & 36004.8 & 135734.7 & 0.3 & 36004.8 & 36004.8 & 17557.4 & 17557.4 & 17189.8 & 88309.4 \\
\hline
\end{tabular}

Table 6: Die design points at different die radius

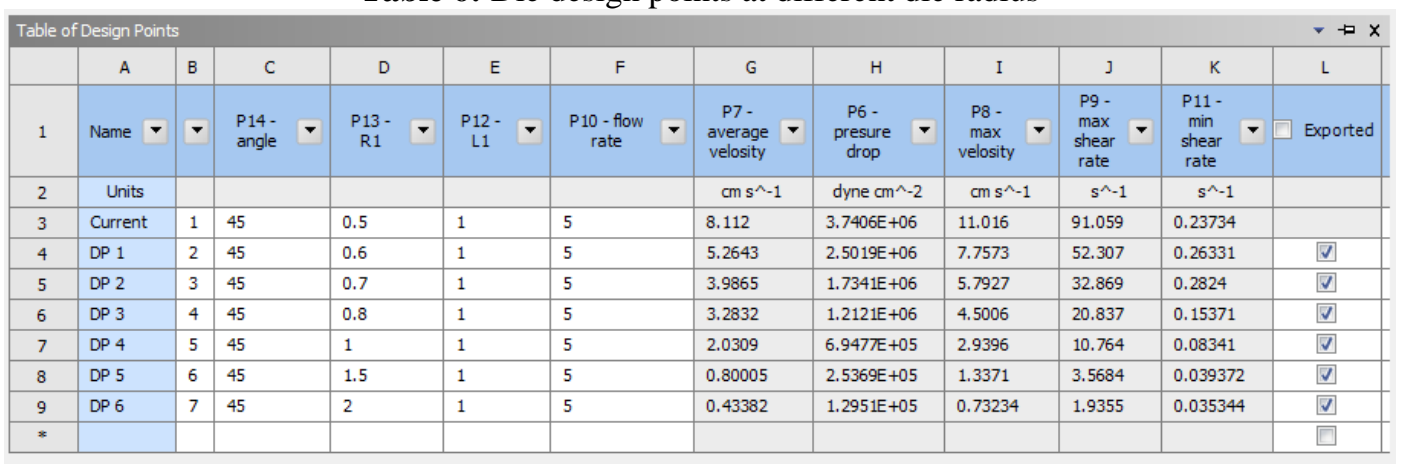




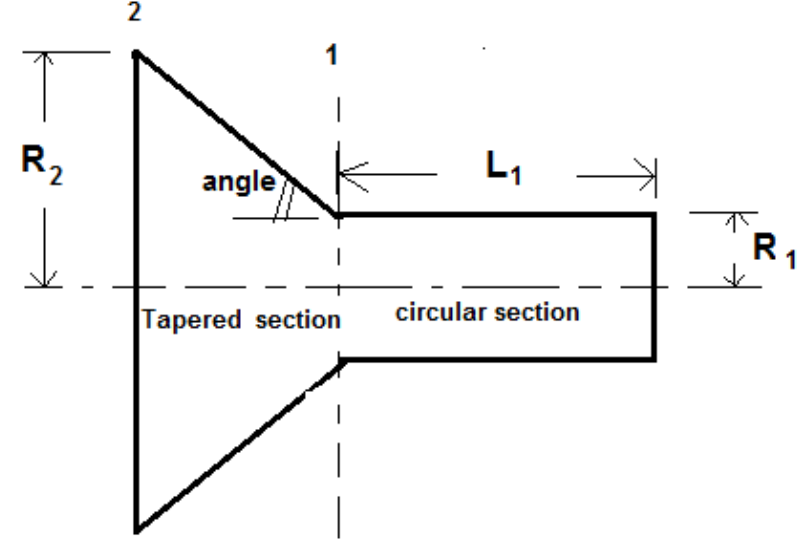

Fig 1: Die section
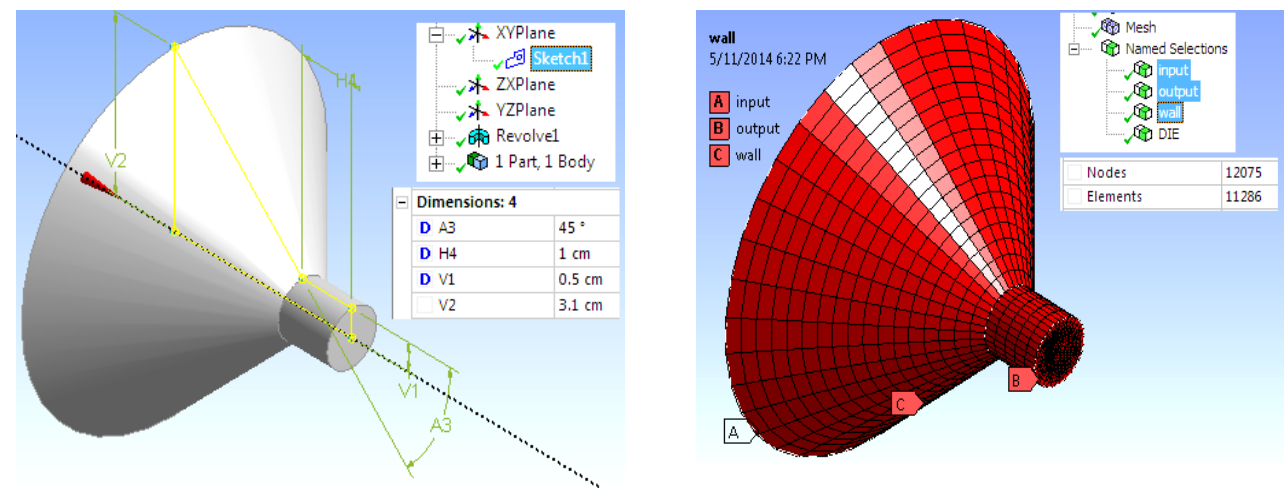

Fig 2: Geometry and Meshing for DIE

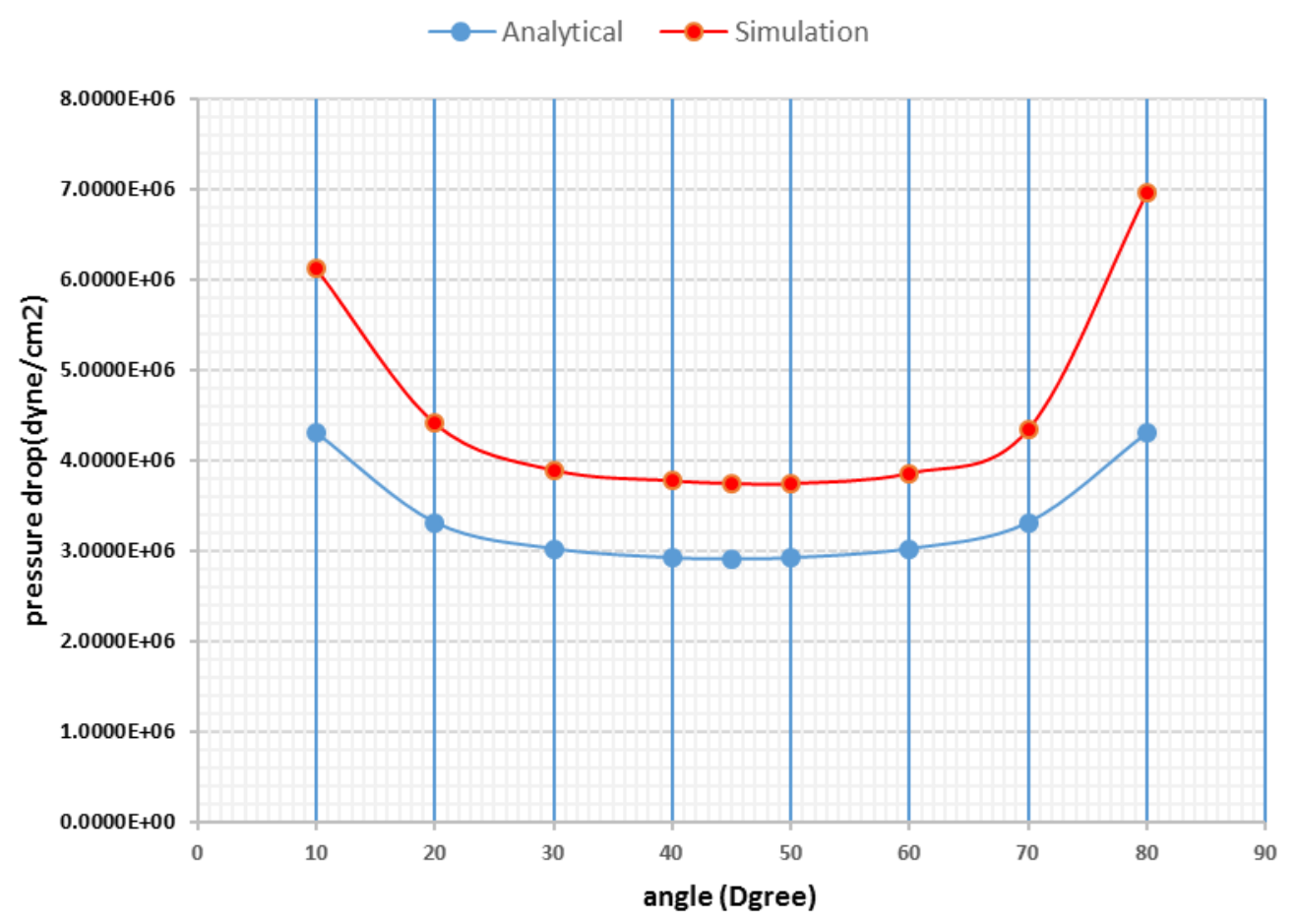

Fig 3: Effect of angle on pressure drop 


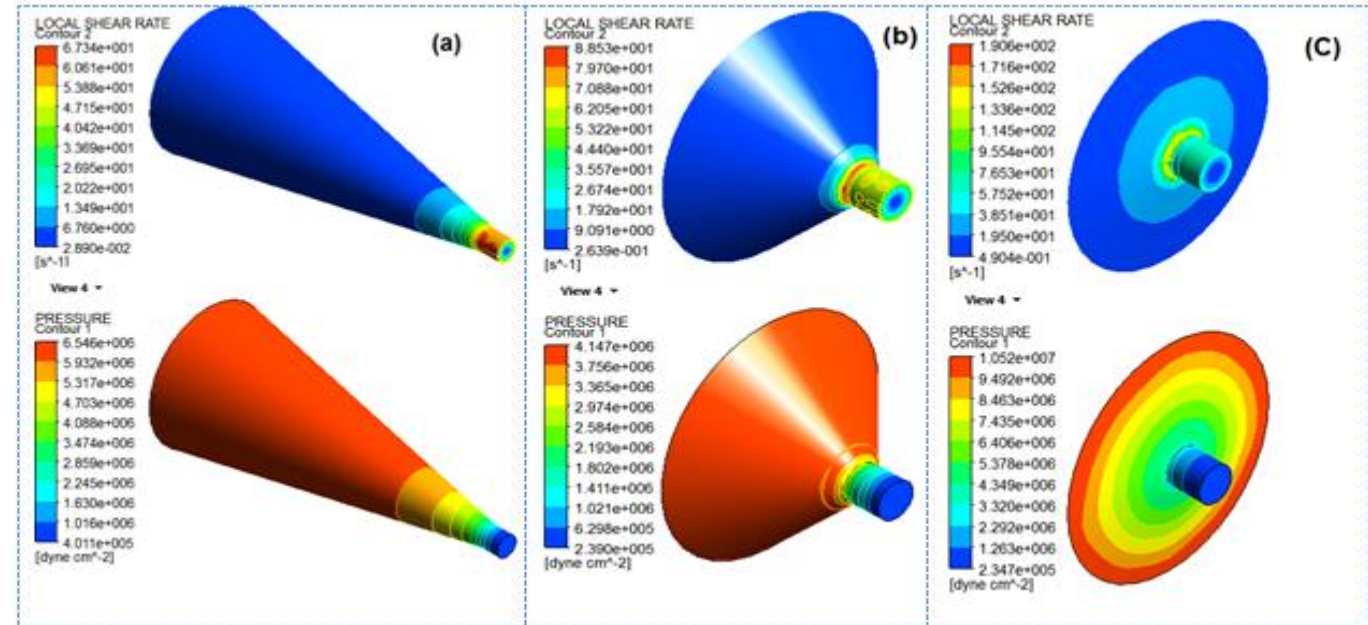

Fig 4: Counters of shear rate and pressure in DIE a) angle $=10$, b) angle $=45$, c) angle $=80$

- $\quad$ analytical simulation $\quad$......... Linear (analytical) $\quad$......... Linear (simulation)

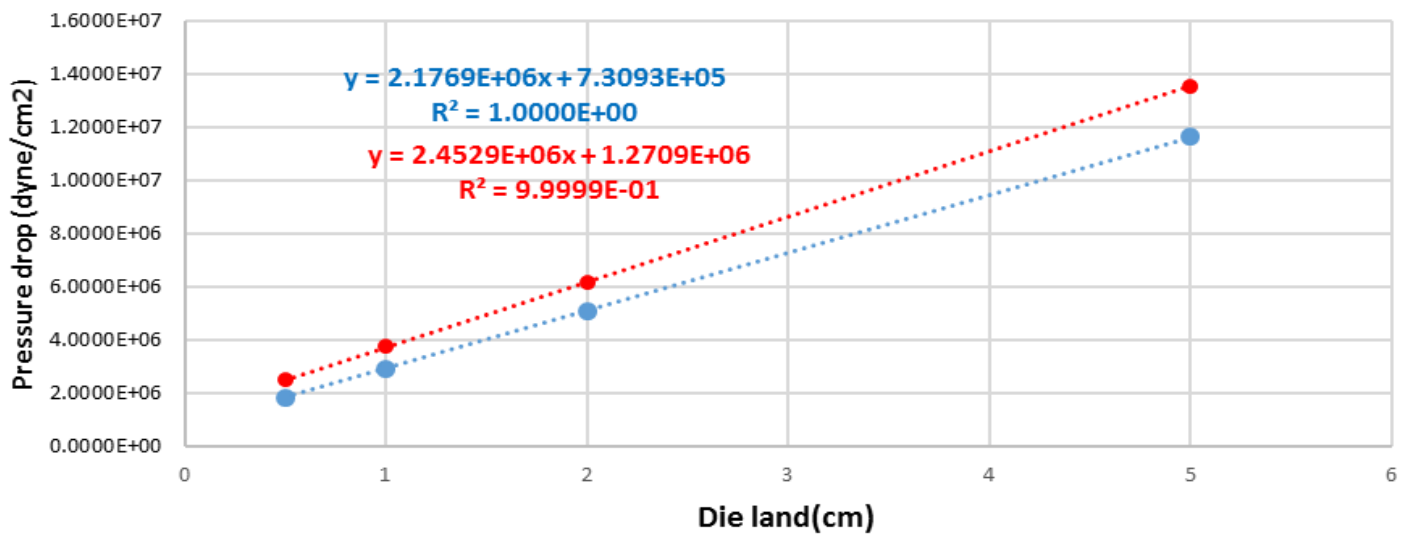

Fig 5: Effect Die land on pressure drop

View 1 .

LOCAL SHEAR RATE

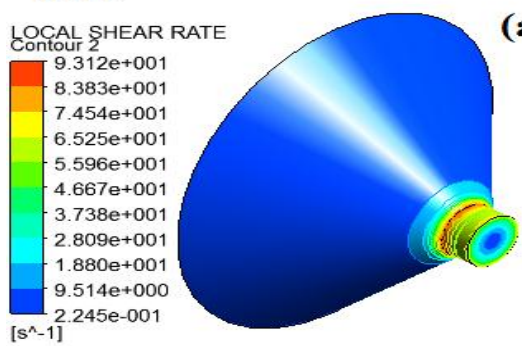

RRESSURE

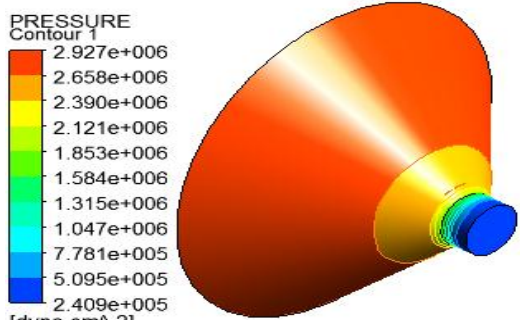

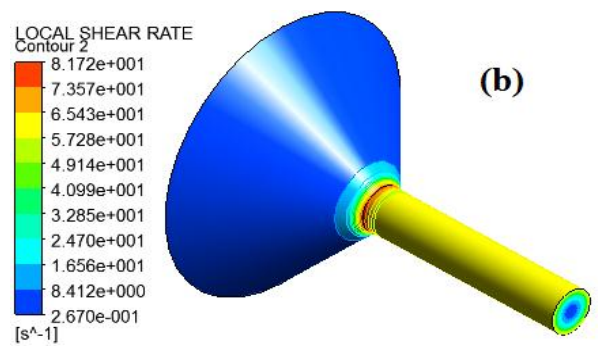

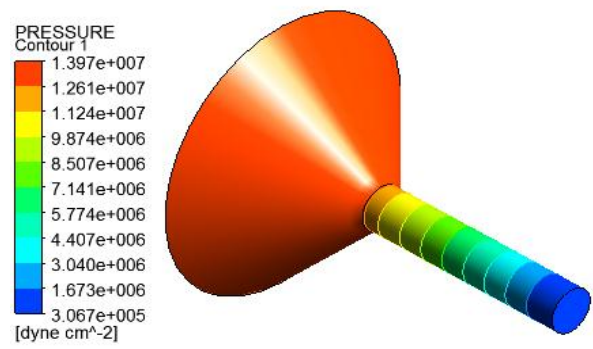

Fig 6: Counters of shear rate and pressure in DIE a) length $=0.5, b)$ length $=5$ 


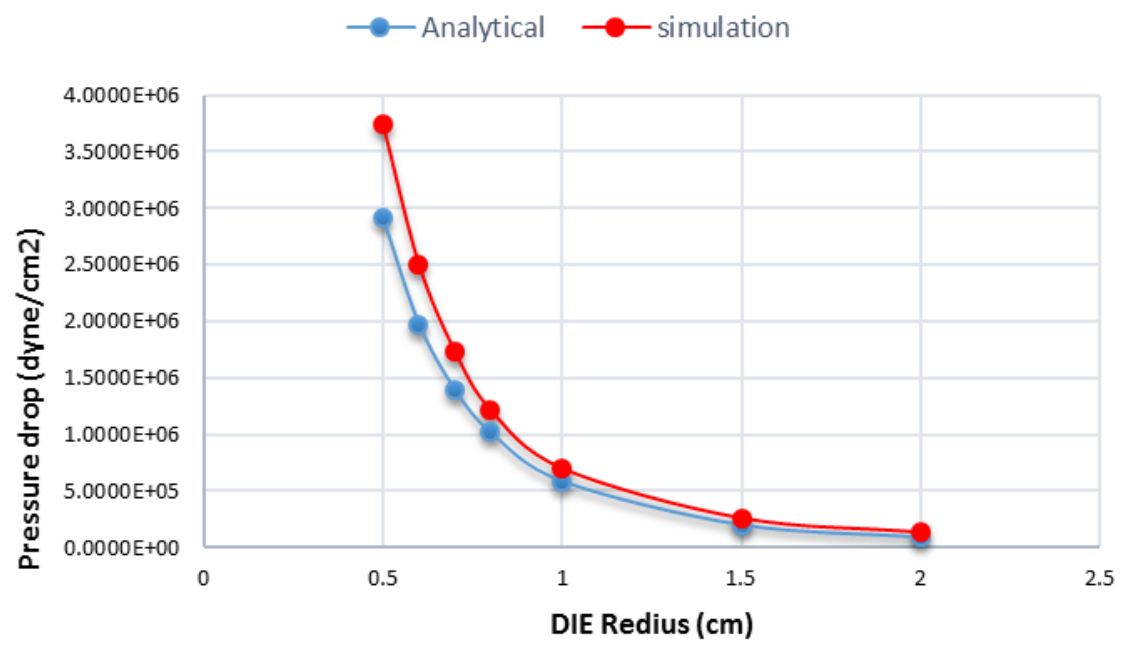

Fig 7: Effect Die radius on pressure drop
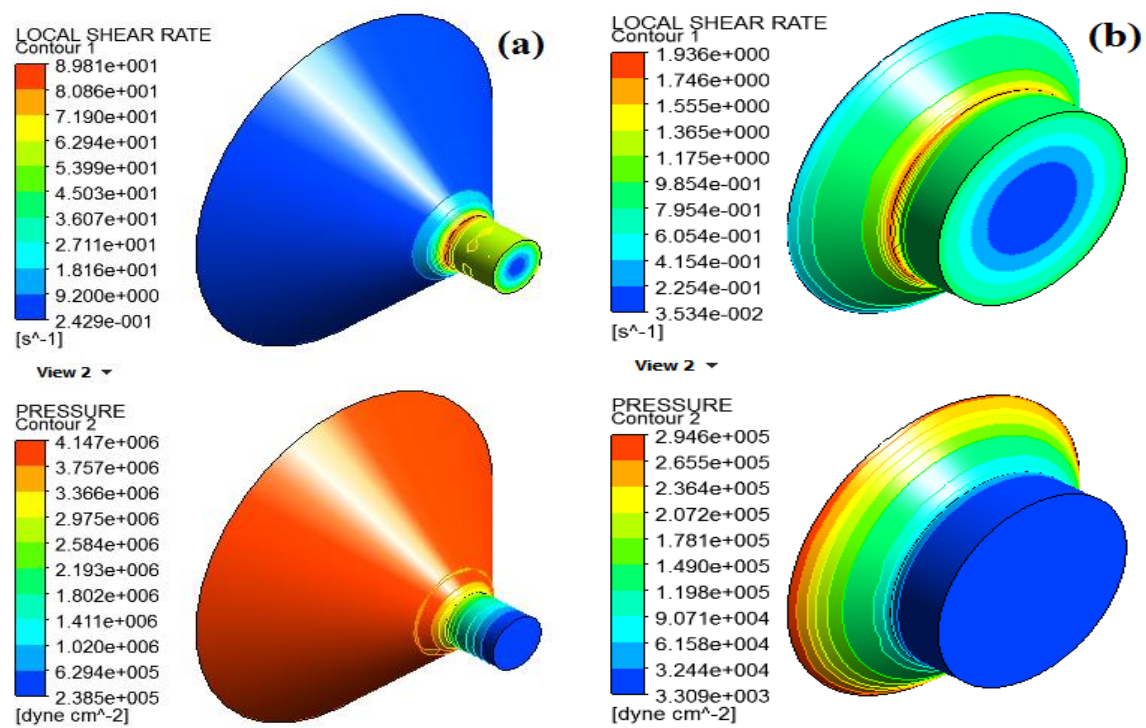

Fig 8: counters of shear rate and pressure in DIE a) radius $=0.5, b$ ) radius $=2$

\section{References}

[1]. R. J. Crawford, Plastics engineering: Butterworth-Heinemann, 1998.

[2]. S. G. Hatzikiriakos and E. Mitsoulis, "Slip effects in tapered dies," Polymer Engineering \& Science, vol. 49, pp. 1960-1969, 2009.

[3]. E. Bagley, "End corrections in the capillary flow of polyethylene," Journal of Applied Physics, vol. 28, pp. 624-627, 2004.

[4]. M. Ansari, A. Alabbas, S. Hatzikiriakos, and E. Mitsoulis, "Entry flow of polyethylene melts in tapered dies," International Polymer Processing, vol. 25, pp. 287-296, 2010.

[5]. E. Mitsoulis, I. B. Kazatchkov, and S. G. Hatzikiriakos, "The effect of slip in the flow of a branched PP melt: experiments and simulations," Rheologica acta, vol. 44, pp. 418-426, 2005.

[6]. M. Kostic and L. Reifschneider, "Design of extrusion dies," Encyclopedia of Chemical Processing DOI, vol. $10,2006$.

[7]. K. Pepliński and A. Mozer, "Design of extrusion die for plastic profile using ansys polyflow software," Journal of Polish CIMAC, vol. 6, pp. 221-226, 2011.

[8]. A. M. a. A.Sidahmed, "Use of Least Square Procedures and Ansys Polyflow Software to Select Best Viscosity Model for Polypropylene," ijesit, vol. 2, November 2013. 